\title{
Indentation failure of circular composite sandwich plates
}

\author{
A. Rajaneesh ${ }^{1}$, I. Sridhar ${ }^{1 *}$, A. R. Akisanya ${ }^{2}$ \\ ${ }^{1}$ School of Mechanical and Aerospace Engineering, Nanyang Technological University, \\ 50 Nanyang Avenue, Singapore 639798, Singapore. \\ ${ }^{2}$ School of Engineering, University of Aberdeen, Aberdeen AB24 3UE, United Kingdom
}

\begin{abstract}
Ski boards, helmets are sandwich structures and prone to core indentation failure under localised loads. In this work, axisymmetric response of a circular composite sandwich plate subjected to indentation by a rigid flat/hemi-spherical punch is examined. Flat punch is assumed to impose an axisymmetric line load, whereas spherical indentor imposes point load. Small deformation response is investigated by solving the equilibrium equations exactly, while large deformation response is estimated using Berger's method. The indentation behavior is predicted numerically by modelling core as (i) a continuum foam and (ii) a plate on foundation with reaction force (i.e. interaction problem) by employing user interaction subroutine in commercial finite element package Abaqus ${ }^{\circledR}$. Derived analytical estimates for the indentation loads and the corresponding finite element predictions are found to be in good agreement with the experimental measurements.

Keywords: Composite Sandwich Plates; Non-linear behavior; Indentation; Flat/Spherical Punch
\end{abstract}

\section{Introduction}

Sandwich construction has gained acceptance as sport sticks [1] and ski boards [2], protective helmet (head-gear) because of their superior specific strength and stiffness compared

\footnotetext{
${ }^{*}$ Corresponding author.

Email address: msridhar@ntu.edu.sg (I. Sridhar ${ }^{1}$ )
} 
to monolithic plates made from either core or faceplate materials. The protective head-gear (helmet) is also a sandwich structure. The core of the sandwich structures is susceptable to indentation failure due to its relatively low modulus and strength when loaded locally. Stress and failure analysis of sandwich structures under quasi-static localized loads and low velocity impact using experimental tests can be expensive while finite element (FE) predictions are computationally intensive. Hence, there is a need for analytical modeling of the deformation and failure behavior of composite sandwich plates for design purposes. The impact of the relevant studies can be seen from the recent works [3-6] in a wide range of applications.

Considerable research has been carried out on the response of sandwich beams and plates subjected to quasi-static indentation loading [7]. Current methodology differs from the higher order sandwich plate theory (HSPT) [8] in which the core is considered to be elastic, while in current study the emphasis is on the post core indentation failure behavior. Several indentation models [7, 9, 12] ignored the bottom faceplate deflection to effectively investigate the local deformation and post indentation failure response in the sandwich structures and these studies have shown reasonable comparison with experiments for circular sandwich plates. These contributions motivate the methodology implemented in the present study. The following discussion is confined only to sandwich plates with composite faceplates, which are usually considered as linear elastic in nature. To investigate the local indentation of the sandwich plates, the core is in general treated as a deformable foundation (viz., elastic; rigid perfectly plastic (RPP); or elastic-perfectly plastic, EPP). Existing indentation failure models from the literature are summarized in Table 1 for flat punch (FP) as well as spherical punch (SP) loading. 
Table 1 Literature on the indentation response of composite sandwich plates.

\begin{tabular}{|c|c|c|c|c|}
\hline Punch & Deformation & Core & Literature & Remarks and assumptions \\
\hline \multirow{13}{*}{$\begin{array}{l}\text { Spherical } \\
\text { (SP) }\end{array}$} & \multirow[t]{7}{*}{ Small } & \multirow[t]{5}{*}{ Elastic } & {$[13]^{\dagger \neq \$ \#}$} & Arbitrary elastic foundations. \\
\hline & & & {$[14,15]^{\dagger \S \#}$} & Strength of materials approach. \\
\hline & & & {$[16]^{\S \ddagger}$} & $\begin{array}{l}\text { Exact solutions in terms of Bessel } \\
\text { functions. }\end{array}$ \\
\hline & & & {$[17]^{\#}$} & $\begin{array}{l}\text { Three dimensional elasticity solu- } \\
\text { tion. Punch loading pressure and } \\
\text { contact radius are variables. }\end{array}$ \\
\hline & & & {$[18]^{\S}$} & $\begin{array}{l}\text { Contact pressure from SP loading is } \\
\text { taken from elastic-half space anal- } \\
\text { ogy. }\end{array}$ \\
\hline & & RPP & {$[11]^{\S}$} & $\begin{array}{l}\text { Solved equilibrium differential equa- } \\
\text { tions in terms of Bessel function. }\end{array}$ \\
\hline & & EPP & {$[11]^{\S}$} & $\begin{array}{l}\text { Modified RPP foundation solution to } \\
\text { achieve EPP foundation response by } \\
\text { adding the elastic limit displacement. }\end{array}$ \\
\hline & \multirow[t]{6}{*}{ Large } & Elastic & {$[19]^{\dagger+\$}$} & Solved using perturbation methods. \\
\hline & & & {$[20]^{\$ \#}$} & $\begin{array}{l}\text { Closed form series solutions were } \\
\text { derived. }\end{array}$ \\
\hline & & RPP & {$[10]^{\text {Hh }}$} & $\begin{array}{l}\text { Bending rigidity and in-plane dis- } \\
\text { placements of the faceplate were ne- } \\
\text { glected. The SP is assumed to be a } \\
\text { FP of } 0.4 \times \text { SP radius. }\end{array}$ \\
\hline & & & {$[11]^{\S}$} & $\begin{array}{l}\text { Simultaneous differential equations } \\
\text { were solved numerically. }\end{array}$ \\
\hline & & & {$[12]^{\S \natural}$} & $\begin{array}{l}\text { Simplistic function was assumed for } \\
\text { in-plane displacement. }\end{array}$ \\
\hline & & EPP & {$[9]^{\dagger \S}$} & $\begin{array}{l}\text { Approximate solution was proposed } \\
\text { by scaling the indentation load from } \\
\text { small deformation theory. }\end{array}$ \\
\hline \multirow[t]{6}{*}{ Flat } & \multirow[t]{3}{*}{ Small } & Elastic & {$[13]^{\ddagger \S},[15]^{\dagger, \ddagger}$} & $\begin{array}{l}\text { Flat punch loading was modelled as } \\
\text { axisymmetric line load. }\end{array}$ \\
\hline & & & {$[18]^{\S}$} & $\begin{array}{l}\text { Contact pressure distributions result- } \\
\text { ing from punch loading were taken } \\
\text { from elastic-half space analogy. }\end{array}$ \\
\hline & & RPP, EPP & & None in literature. \\
\hline & \multirow[t]{3}{*}{ Large } & Elastic & {$[19]^{\ddagger \S},[15]^{\dagger, \ddagger}$} & $\begin{array}{l}\text { Flat punch loading was modelled as } \\
\text { axisymmetric line load. }\end{array}$ \\
\hline & & RPP & {$[21]^{\S ‡}$} & $\begin{array}{l}\text { Extended analysis in [10] to arbitrary } \\
\text { faceplate composite layup. }\end{array}$ \\
\hline & & EPP & & None in literature. \\
\hline
\end{tabular}


The governing differential equations of equilibrium resulting from small deformations can be solved exactly. However, incorporating large deformations to the faceplate leads to two simultaneous coupled differential equations [15, 19] which can not be solved exactly. In estimating the large deformation behavior analytically, one could adopt to: (i) approximate methods, e.g., Ritz method, Galerkin's method, Perturbation methods, Fourier series solutions [10, 12, 19, 20], (ii) scaling the load estimated from the small deformation theory using membrane stiffness [9], (iii) solving the differential equations numerically [11] or (iv) uncoupling the differential equations with appropriate assumptions as stated in [22]. Sandwich plates with metallic faceplates which are elastic-plastic need a different methodology (due to formation of plastic hinges on the faceplate) as described in [23, 24] and are not considered in the current study.

Most of the existing studies focused on the indention of sandwiched plates subjected to spherical punch (SP) loading, in which the faceplate in-plane displacements were neglected. Studies on the indentation behavior of sandwich plates with flat punch (FP) loading are limited [21]. None of the contributions in open literature, e.g. [9, 11] provides a closed form solution, that considers the midplane displacements of the faceplate when subject to indentation loading. However, a few existing closed form solutions for load-displacement response [10, 12, 21] are based on too-simplistic assumptions of either neglecting midplane displacements of the faceplate or based on polynomial approximations for the displacement.

Hence in the current work, small deformation solutions for a sandwich structure subject to indentation loading are derived by exactly solving the corresponding differential equations for a flat punch (FP). Then, analytical solutions resulting from large deformations are derived for the indentation of circular composite sandwich plates by a FP including radial inplane displacement using Berger's method [22]. Subsequently, the analytical solution of the deformation of sandwich plate subjected to indentation load by a spherical punch (SP) is derived from the corresponding solution of FP [14]. 


\section{Analytical modeling}

To investigate the indentation response of a sandwich plate subjected to localized loads, the global bending of the sandwich plate is avoided by considering a sandwich plate resting on a rigid backing as shown in Figure 1a Consider a circular sandwich plate with a radius $R$, consisting of a core of thickness $c$, bonded between two identical elastic faceplates each of thickness $t$ and subjected to indentation load via a rigid flat indenter of radius $a$. The Young's modulus, Poisson's ratio and compressive strength of the core are $E_{c}, v_{c}$ and $\sigma_{c}$, respectively. The faceplates are made from a material with Young's modulus $E_{f}$, Poisson's ratio $v_{f}$ and equivalent bending rigidity $D_{f}$ expressed as [9]: $D_{f} \approx \sqrt{D_{11} D_{22}(\eta+1) / 2}$ where $\eta=$ $\left(D_{12}+2 D_{16}\right) / \sqrt{D_{11} D_{22}}$ and $D_{i j}$ are the components of the bending stiffness matrix. For an isotropic plate $D_{f} \approx E_{f} t^{3} /\left[12\left(1-v_{f}^{2}\right)\right]$. A polar coordinate system $r-z$ is defined at the intersection of the longitudinal axis of the punch and the mid surface of the top faceplate of the sandwich plate. It is assumed that the radius of the plate is much larger than the punch radius, i.e., $R \gg a$ and the core thickness is much larger than the faceplate thickness $(c>>t)$. Hence, $R$ and $c$ have negligible effect on the overall local indentation behavior. The deformed shape of the faceplate does not conform to the punch shape after the initial deformation [25]. Hence, the applied indentation load $P$ is distributed over the circumference of a circle of radius $a$ as an axisymmetric line load with magnitude $p=P /(2 \pi a)$. Consequently, the contact interaction between the faceplate and the indenter in the region $0 \leq r \leq a$ is not considered in the analytical modeling. The central displacement corresponding to the punch load $P$ is $\delta$.

\subsection{Small deformation of a plate resting on an EPP foundation}

The purpose of the analysis presented in this section is to determine three important aspects of an indentation problem: (i) the load-displacement response of the indentor, (ii) the local deformed profile of the top faceplate when subjected to an arbitrary indentation load $P=2 \pi a p$ and (iii) the load versus plastic radius relationship. 


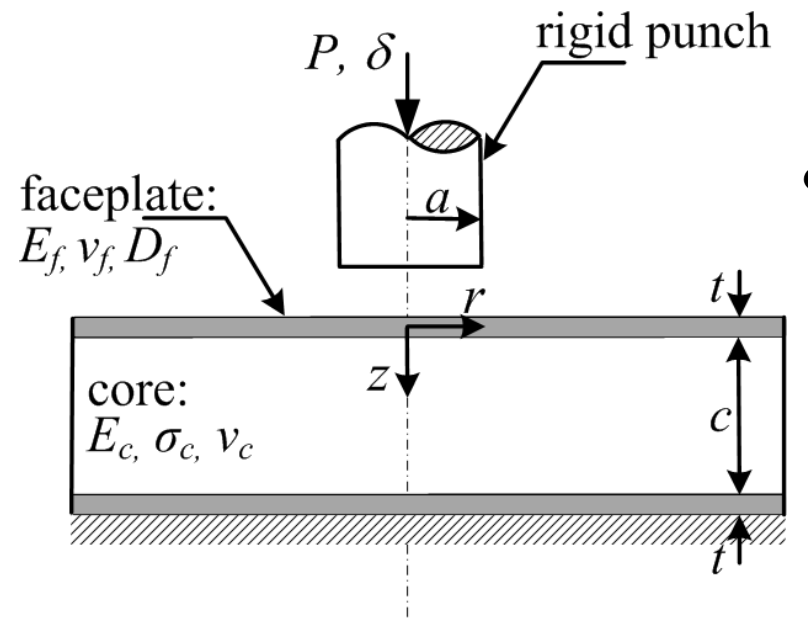

(a) Sandwich plate on rigid base.
Axisymmetric line load of intensity, $p=P /(2 \pi a)$

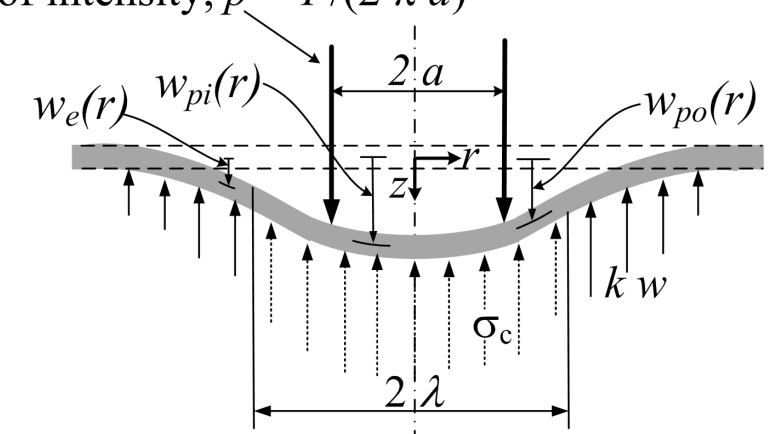

(b) Small deformation (FP): EPP

Axisymmetric line load of intensity, $p=P /(2 \pi a)$

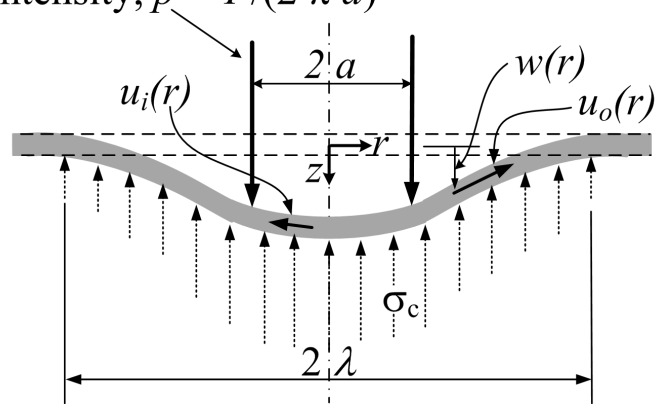

(c) Large deformation (FP): RPP

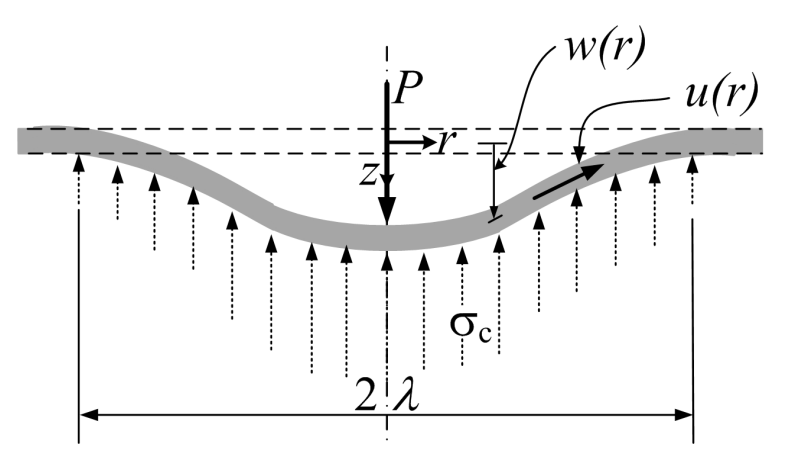

(d) Large deformation (SP): RPP

Figure 1 Schematic representation of a sandwich plate subjected to indentation loading.

At the start of the indentation, the core behaves as elastic foundation. The deformation behavior of a plate on an elastic foundation is given by Panc [26] as

$$
\begin{aligned}
& w_{i}(r)=\frac{P l^{2}}{4 D_{f}}\left[\mathrm{f}_{0}(a) \mathrm{u}_{0}(r)-\mathrm{g}_{0}(a) \mathrm{v}_{0}(r)\right] ; \\
& w_{o}(r)=\frac{P l^{2}}{4 D_{f}}\left[\mathrm{u}_{0}(a) \mathrm{f}_{0}(r)-\mathrm{v}_{0}(a) \mathrm{g}_{0}(r)\right]
\end{aligned}
$$

where, $w_{i}$ and $w_{o}$ are the deflection profiles of the top faceplate inside and outside the loading radius $a$, respectively, $l\left(=\sqrt[4]{D_{f} / k}\right)$ is a characteristic length, $k$ is the foundation modulus (defined in Eq. (2)), $\mathrm{u}_{n}(r)=\operatorname{Re}_{n}\left(r \mathrm{e}^{i \psi} / l\right), \mathrm{v}_{n}(r)=\operatorname{Im~J}_{n}\left(r \mathrm{e}^{i \psi} / l\right), \mathrm{f}_{n}(r)=\operatorname{Re}_{n}^{(1)}\left(r \mathrm{e}^{i \psi} / l\right)$, and $\mathrm{g}_{n}(r)=\operatorname{Im~H}_{n}^{(1)}\left(r \mathrm{e}^{i \psi} / l\right)$ are real valued functions, $\mathrm{J}_{n}$ is the Bessel's function of first kind of $n^{\text {th }}$ index, $\mathrm{H}_{n}^{(1)}, \mathrm{H}_{n}^{(2)}$ are the first and second Hankel's functions of $n^{\text {th }}$ index, respectively and $\psi=\pi / 4$. Conventionally, foundation modulus is defined as $k=E_{c} / c$. However, as the 
core thickness tends to infinity $(c \rightarrow \infty)$, the foundation modulus tends to zero $(k \rightarrow 0)$, which is physically unrealistic. Hence, to limit the value of the $k$ for thick core, the following limits are used [9]:

$$
k=\frac{E_{c}}{c^{*}\left(1-v_{c}^{2}\right)} ; \quad \text { where } \quad c^{*}=\min \left(c, c^{3 D}\right) ; \quad c^{3 D}=\frac{128}{27}\left[\frac{2 D_{f}\left(1-v_{c}^{2}\right)}{E_{c}}\right]^{1 / 3}
$$

As the indentation progresses, the core starts to yield when the indentation load and the corresponding punch displacement reaches their limiting values (according to maximum throughthickness stress criterion) [25]:

$$
\delta_{e l}=\frac{\sigma_{c}}{k} ; \quad P_{e l}^{F P}=\frac{4 D_{f} \sigma_{c}}{k l^{2}} \frac{1}{\mathrm{u}_{0}(a) \mathrm{f}_{0}(a)-\mathrm{v}_{0}(a) \mathrm{g}_{0}(a)}
$$

For load, $P \geq P_{e l}^{F P}$ or punch displacement, $\delta \geq \delta_{e l}$, the core yields and behaves as an EPP foundation as shown in Figure $1 \mathrm{~b}$, in which $\lambda$ is the plastic radius (equals to $a$ at the onset of plastic yield). For an indentation load $P \geq P_{e l}^{F P}$, the plastic zone $\lambda$ will propagate outward beyond the punch radius $a$ i.e. $\lambda>a$. Within the plastic zone $0 \leq r \leq \lambda$, the core has been compressed to its yield strength and thus exerts a uniform pressure of magnitude $\sigma_{c}$ on the top faceplate. However, in the region $r \geq \lambda$, the deformation of the core is still elastic and the core exerts a load of magnitude $k w$ on the top faceplate. For simplicity, we divide the deformation region into three zones: (i) the plastic zone in the interval $0 \leq r \leq$ $a$ with transverse displacement $w_{p_{i}}$, (ii) the plastic zone in the $a \leq r \leq \lambda$ with transverse displacement designated as $w_{p_{o}}$, and (iii) the elastic zone in the interval $\lambda \leq r \leq \infty$ with a transverse displacement denoted by $w_{e}$. The governing differential equations for the three zones are given respectively by

$$
\begin{array}{ll}
\text { for } 0 \leq r \leq a: & D_{f} \nabla_{r}^{2} \nabla_{r}^{2} w_{p_{i}}=-\sigma_{c} \\
\text { for } a \leq r \leq \lambda: & D_{f} \nabla_{r}^{2} \nabla_{r}^{2} w_{p_{o}}=-\sigma_{c} \\
\text { for } \quad r \geq \lambda: & D_{f} \nabla_{r}^{2} \nabla_{r}^{2} w_{e}+k w_{e}=0
\end{array}
$$

where $\nabla_{r}^{2}$ is the Laplacian operator in polar coordinates. Ensuring finite deflection and zero slope at $r=0$ and vanishing deflection at $r=\infty$, the general solutions to Eq. (4) are 
shown as

$$
\begin{array}{ll}
\text { for } 0 \leq r \leq a: & w_{p_{i}}(r)=B_{1} r^{2}+B_{2}-\frac{\sigma_{c} r^{4}}{64 D_{f}} \\
\text { for } a \leq r \leq \lambda: & w_{p_{o}}(r)=B_{3} r^{2} \ln (r)+B_{4} \ln (r)+B_{5} r^{2}+B_{6}-\frac{\sigma_{c} r^{4}}{64 D_{f}} \\
\text { for } \quad r \geq \lambda: & w_{e}(r)=B_{7} \mathrm{f}_{0}(r)+B_{8} \mathrm{~g}_{0}(r)
\end{array}
$$

where $B_{j}(j=1$ to 8$)$ are the integration constants determined from the continuity in transverse displacement, slope, bending moment and shear force at $r=a$ and $r=\lambda$, and the derived constants $B_{j}$ are given in Appendix A

Additionally, the unknown load $P$ versus indentation radius $\lambda$ relationship is established using the condition $w_{p_{o}}(\lambda)=\sigma_{c} / k$ to obtain

$$
\begin{aligned}
P=\frac{\pi}{2 \eta_{12}}\{8 & \sqrt{2} l^{2} \eta_{7}\left(\sigma_{c} \lambda^{2}-2 \eta_{11}\right)-\sqrt{2} \eta_{8}\left[\sigma_{c} \lambda^{4}-2 \frac{P_{e l}^{i}}{\pi}\left(\lambda^{2}-a^{2}\right)\right] \\
& \left.+16 \lambda l\left(\eta_{6} \eta_{11}-2 \sigma_{c} l^{2} \eta_{5}\right)\right\}
\end{aligned}
$$

where expressions for $\eta_{j}(j=1$ to 13) are given in Appendix A.

The above solution can be used to analyse the small deformation of a sandwich plate subjected to localized indentation. For a given plastic radius, $\lambda$, the indentation load $P$ is estimated from Eq. (6), and the corresponding punch displacement $\delta=w_{p_{i}}(a)$ is found from Eq. (5a). To establish the load-displacement response: a range is assumed for plastic radius e.g. $\lambda=a$ to $2.5 a$ and for each value of $\lambda$, Eq. (5a) at $r=a$ and Eq. (6) are evaluated to obtain the corresponding punch displacement and indention load, respectively.

To establish the deformation profile of the top faceplate at a plastic radius, $\lambda$; the indentation load, $P$ obtained from Eq. (6) is used to evaluate the deformation profile from Eqs. (5a) -(5c) for a range of $r$ values of interest e.g. $r=0$ to $2.5 a$.

\subsection{Large deformation theory}

In this section, large deformation of the faceplate is considered using the approximate differential equations given by Berger [22]. Initially the load-displacement relations are derived for FP loading and these are subsequently modified by making the punch radius $a=0$ to get 
the corresponding solutions for the spherical punch.

\subsubsection{Plate on RPP foundation subjected to FP loading}

Berger [22] assumed that the second invariant of midplane strains can be neglected without loss of much accuracy in predicting the large deformation behavior. This assumption led to two uncoupled differential equations governing in-plane $(u)$ and out-of-plane $(w)$ displacements and hence can be solved individually. The uncoupled governing differential equations for FP loading can be expressed as:

$$
\begin{aligned}
\frac{\mathrm{d}}{\mathrm{d} r}\left(\nabla_{r}^{2} w\right)-\alpha^{2} \frac{\mathrm{d} w}{\mathrm{~d} r} & =-\frac{\sigma_{c} r}{2 D_{f}}+\frac{P \mathcal{H}(r-a)}{2 \pi r D_{f}} \\
\frac{1}{r} \frac{\mathrm{d}}{\mathrm{d} r}(r u) & =\frac{\alpha^{2} t^{2}}{12}-\frac{1}{2}\left(\frac{\mathrm{d} w}{\mathrm{~d} r}\right)^{2}
\end{aligned}
$$

where, $\alpha$ is the Berger's constant, $\mathcal{H}(r-a)$ is the Heaviside step function equals to zero if $r<a$ and one if $r \geq a$. Equation (7a) provides the bending deflection while Eq. (7b) governs the stretching deformation of the faceplate. The general solution to the Eq. (7a), after imposing finite deflection and zero slope at $r=0$ can be shown to be

$$
w(r, \alpha)=C_{1} \mathrm{I}_{0}(\alpha r)+C_{2}+\frac{\sigma_{c} r^{2}}{4 D_{f} \alpha^{2}}+\frac{P \mathcal{H}(r-a)}{2 \pi D_{f} \alpha^{2}}\left[\Upsilon_{1}-\ln \left(\frac{r}{a}\right)\right]
$$

where, $C_{i}$ are the integration constants to be determined from boundary conditions at $r=\lambda, \mathrm{I}_{n}$ is the modified Bessel's function of first kind of $n^{\text {th }}$ index and $\Upsilon_{i}$ are defined in Appendix B.

Expanding the Heaviside function in Eq. (8) and substituting it in Eq. (7b) and successive integration gives the in-plane displacement functions $u_{i}$ and $u_{o}$ for the inside and outside the loading radius respectively, as

$$
\begin{aligned}
& u_{i}(r, \alpha)=\frac{\alpha^{2} t^{2} r}{24}+\frac{C_{3}}{r}-\left[\frac{C_{1}^{2} \alpha^{2} \Upsilon_{3} r}{4}+\frac{C_{1} r \sigma_{c} \mathrm{I}_{2}(\alpha r)}{2 \alpha^{2} D_{f}}+\frac{\sigma_{c}^{2} r^{3}}{32 D_{f}^{2} \alpha^{4}}\right] \\
& u_{o}(r, \alpha)=\frac{\alpha^{2} t^{2} r}{24}+\frac{C_{4}}{r}-\left\{\frac{C_{1}^{2} \alpha^{2} \Upsilon_{3} r}{4}+\frac{C_{1} r \sigma_{c} \mathrm{I}_{2}(\alpha r)}{2 \alpha^{2} D_{f}}+\frac{\sigma_{c}^{2} r^{3}}{32 D_{f}^{2} \alpha^{4}}\right. \\
& \left.+P^{2}\left[\frac{\mathrm{K}_{0}(\alpha a) \mathrm{I}_{0}(\alpha a) \Upsilon_{4}}{16 \sqrt{\pi^{5}} D_{f}^{2} \alpha^{2}}+\Upsilon_{6}\right]-\frac{P \sigma_{c} r\left(1+2 \Upsilon_{5}\right)}{8 \pi D_{f}^{2} \alpha^{4}}+P\left[\frac{C_{1} \mathrm{I}_{0}(\alpha a) \Upsilon_{4}}{8 \sqrt{\pi^{3}} D_{f}}+\Upsilon_{7}\right]\right\}
\end{aligned}
$$

where $C_{3}$ and $C_{4}$ are the integration constants and $\mathrm{K}_{n}$ is the modified Bessel's function 
of second kind of $n^{\text {th }}$ index, functions $\Upsilon_{i}$ are provided in Appendix B. Since the in-plane displacement is zero at the center of the plate i.e. $u_{i}(0)=0, C_{3}$ becomes zero. The integration constant $C_{4}$ is calculated using the continuity condition $u_{i}(a)=u_{o}(a)$. Additional unknown load versus plastic radius, $P-\lambda$, relation is found using the zero radial moment condition i.e. $M_{r}(\lambda)=0$ and can be shown to be

$$
P(\alpha)=\frac{\pi \lambda \sigma_{c}}{\alpha} \frac{2 \mathrm{I}_{1}(\alpha \lambda)-\alpha \lambda \mathrm{I}_{0}(\alpha \lambda)}{\mathrm{I}_{0}(\alpha a)-\mathrm{I}_{0}(\alpha \lambda)}
$$

The unknown Berger's constant, $\alpha$ can only be found numerically, by solving $u_{o}(\lambda)=0$. With this, the analytical model for the large deformation of a sandwich plate subjected to FP loading is obtained as follows: for a given $\lambda(>0)$, the indentation load $P(\alpha)$ from Eq. (10) is substituted into Eq. (9b) to numerically solve for $\alpha$ using $u_{o}(\lambda)=0$ boundary condition. The corresponding punch displacement $\delta(\alpha)=w(0)$ is estimated from Eq. (8).

In the limiting case as $\alpha \rightarrow 0$, Eqs. (8)-(10) reduce to the following small deformation solutions

$$
\begin{aligned}
w(r) & =\frac{P \mathcal{H}(r-a)}{8 \pi D_{f}}\left[\left(r^{2}+a^{2}\right) \ln \left(\frac{r}{a}\right)+a^{2}-r^{2}\right]-\frac{\sigma_{c} r^{4}}{64 D_{f}}+\frac{C_{1}^{\prime} r^{2}}{4}+C_{2}^{\prime} \\
P & =\frac{\pi \lambda^{4} \sigma_{c}}{2\left(\lambda^{2}-a^{2}\right)}
\end{aligned}
$$

where

$$
\begin{aligned}
& C_{1}^{\prime}=\frac{P}{4 \pi D_{f}}\left[1-\frac{a^{2}}{\lambda^{2}}-2 \ln \left(\frac{\lambda}{a}\right)\right]+\frac{\sigma_{c} \lambda^{2}}{8 D_{f}} \\
& C_{2}^{\prime}=\frac{P \lambda^{2}}{16 \pi D_{f}}\left[1-\frac{a^{2}}{\lambda^{2}}-2 \frac{a^{2}}{\lambda^{2}} \ln \left(\frac{\lambda}{a}\right)\right]-\frac{\sigma_{c} \lambda^{4}}{64 D_{f}}
\end{aligned}
$$

The solution for a sandwich plate resting on a RPP foundation subject to FP loading is thus established. To estimate the solution for the plate resting on a EPP foundation from the derived RPP foundation solution, a correction to the punch displacement is applied by adding an elastic limit displacement $\delta_{e l}$.

\subsubsection{Plate on RPP foundation with SP loading}

In pratice the contact radius of the spherical punch increases with respect to increase in 
indentation depth. However in the present work effect of contact radius is eliminated based on the assumption: SP is assumed to impose a point load [10, 11]. As mentioned earlier, the solution for SP is derived for a central concentrated load, $P$ by making flat punch radius, $a$ to zero as done by [14]. The assumed point load can be viewed as the static resultant contact force between the SP and the faceplate. The difference between the solution based on the point load and that for the SP which incorporate the contact radius should, according to Saint-Vennat principles, become very small at sufficiently large distance from the point load.

The governing differential equations for SP loading were not derived explicitly, instead the limit $a \rightarrow 0$ is applied to Eqs. (8)-(10). Using the limiting forms $\mathrm{I}_{0} \approx 1, \mathrm{I}_{1} \approx \alpha r / 2$ and $\mathrm{I}_{2}(0) \approx \alpha^{2} r^{2} / 8$ and neglecting all the singular terms resulting from $\mathrm{K}_{n}(\alpha a)$ gives

$$
\begin{aligned}
w(r, \alpha) & =F_{1} \mathrm{I}_{0}(\alpha r)+F_{2}+\frac{\sigma_{c} r^{2}}{4 D_{f} \alpha^{2}}-\frac{P}{2 \pi D_{f} \alpha^{2}}\left[\mathrm{~K}_{0}(\alpha r)+\ln (r)\right] \\
w(0, \alpha) & =F_{1}+F_{2}+\frac{P}{2 \pi D_{f} \alpha^{2}}\left[\ln \left(\frac{2}{\alpha}\right)-\gamma\right] \\
u(r, \alpha) & =\frac{\alpha^{2} t^{2} r}{24}+\frac{F_{3}}{r}-\left\{\frac{F_{1}^{2} \alpha^{2} \Upsilon_{3}^{\prime} r}{4}+\frac{F_{1} r \sigma_{c} \mathrm{I}_{2}(\alpha r)}{2 \alpha^{2} D_{f}}+\frac{\sigma_{c}^{2} r^{3}}{32 D_{f}^{2} \alpha^{4}}+P^{2} \Upsilon_{6}^{\prime}-\frac{P \sigma_{c} r\left(1+2 \Upsilon_{5}^{\prime}\right)}{8 \pi D_{f}^{2} \alpha^{4}}\right. \\
& \left.+P\left[\frac{F_{1} \Upsilon_{4}^{\prime}}{8 \sqrt{\pi^{3}} D_{f}}+\Upsilon_{7}^{\prime}\right]\right\} \\
P(\alpha) & =\frac{\pi \lambda \sigma_{c}}{\alpha} \frac{2 \mathrm{I}_{1}(\alpha \lambda)-\alpha \lambda \mathrm{I}_{0}(\alpha \lambda)}{1-\mathrm{I}_{0}(\alpha \lambda)}
\end{aligned}
$$

where $\gamma(=0.577)$ is the Euler's constant and the integration constants $F_{i}, \Gamma_{1}^{\prime}$ and $\Upsilon_{i}^{\prime}$ are defined in Appendix C. The unknown Berger's constant can only be found numerically by solving $u(\lambda)=0$ boundary condition. With this, analytical model for the large deformation of a sandwich plate subjected to SP loading is studied as follows: for a given $\lambda(>0)$, the indentation load $P(\alpha)$ from Eq. $13 \mathrm{~d}$ ) is substituted into Eq. $13 \mathrm{c}$ ) to numerically solve for $\alpha$ using $u(\lambda)=0$. The corresponding punch displacement $\delta(\alpha)=w(0)$ is estimated from Eq. (13b).

In the limiting case as $\alpha \rightarrow 0$, Berger's solution is found to agree with the small deforma- 
tion equilibrium solution as

$$
\begin{aligned}
w(r) & =\frac{P}{16 \pi D_{f}}\left[2 r^{2} \ln \left(\frac{r}{\lambda}\right)+\lambda^{2}-r^{2}\right]-\frac{\sigma_{c} \lambda^{4}}{64 D_{f}}\left(1-\frac{r^{2}}{\lambda^{2}}\right)^{2} \\
P & =\frac{\pi \lambda^{2} \sigma_{c}}{2}
\end{aligned}
$$

However, to apply the derived RPP foundation solution to EPP foundation, one has to offset the punch displacement by elastic limit displacement $\delta_{e l}=\sigma_{c} / k$ corresponds to a limiting load of $P_{e l}^{S P}=8 \sigma_{c} \sqrt{D_{f} / k}$.

\section{Experimental studies}

This section describes the experimental methodology used to validate the analytical solution presented above. Closed cell Divinycell ${ }^{\circledR}$ poly-vinyl chloride (PVC) foams (supplied by Diab Inc. Thailand) with nominal densities $35 \mathrm{~kg} / \mathrm{m}^{3}$ (H35), $45 \mathrm{~kg} / \mathrm{m}^{3}$ (H45), $80 \mathrm{~kg} / \mathrm{m}^{3}$ (H80) and $100 \mathrm{~kg} / \mathrm{m}^{3}$ (H100) were used as core materials for sandwich plate construction. Uniaxial compression experiments were performed under displacement control at $1 \mathrm{~mm} / \mathrm{min}$ using the specimens of $50 \mathrm{~mm} \times 50 \mathrm{~mm}$ in the raise direction of thicknes. The engineering stress-strain response resembles that of an elastic perfectly-plastic (EPP) behavior and the measure properties are listed in Table 2 .

Table 2 Properties of foams

\begin{tabular}{lcccc}
\hline & \multicolumn{4}{c}{ Foams* } \\
\cline { 2 - 5 } Property & $\mathrm{H} 35$ & $\mathrm{H} 45$ & $\mathrm{H} 80$ & $\mathrm{H} 100$ \\
\hline Density $\left(\mathrm{kg} / \mathrm{m}^{3}\right)$ & 35 & 45 & 80 & 100 \\
Young's Modulus (MPa) & 22 & 35 & 49 & 52 \\
Yield strength (MPa) & 0.5 & 0.65 & 1.2 & 2 \\
Yield strain (\%) & 2.273 & 1.857 & 2.449 & 3.846 \\
\hline
\end{tabular}

* For all the foams an elastic Poisson's ratio of 0.3, plastic Poisson's ratio of 0.0 are used.

Quasi-isotropic composite faceplates with [-60/0/60 $]_{n s}(n=1,2)$ configuration was cured from unidirectional E-glass/epoxy prepregs (supplied by Weihai Guangwei Composites Co., Ltd., China). Thickness of quasi-isotropic laminates $[-60 / 0 / 60]_{1 s}$ and $[-60 / 0 / 60]_{2 s}$ are measured to be $0.7 \mathrm{~mm}$ and $1.3 \mathrm{~mm}$, respectively. The in-plane Young's modulus and Poisson's 
ratio of the quasi-isotropic laminates as reported in our previous work are 25.3 GPa and 0.24, respectively [25].

Sandwich plates were constructed by bonding the quasi-isotropic laminates to the foam core using Hysol EA 9309.3NA epoxy paste adhesive (manufactured by Henkel). Two to four layers of foam blanks were bonded to construct a nominal core of thickness $50 \mathrm{~mm}$ and cured under a pressure of $1 \mathrm{kPa}$ for four days. A faceplate was bonded to the foam core only on the indentation side of the sandwich plate.

The sandwich plate (of $200 \mathrm{~mm}$ x $200 \mathrm{~mm}$ ) was clamped using two mild steel plates (of size $300 \mathrm{~mm} \times 300 \mathrm{~mm}$ using a central circular hole of $160 \mathrm{~mm}$ diameter) on the top and bottom to get a circular plate geometry in experiments. Indentation experiments were conducted with a flat punch of diameter $20 \mathrm{~mm}$ and a spherical punch of diameter $10 \mathrm{~mm}$ by placing the sandwich plate on a flat rigid base. All the indentation experiments were conducted on Instron 5567 under displacement control at a nominal speed of $1 \mathrm{~mm} / \mathrm{min}$.

\section{Finite element modeling}

The accuracy of the analytical models for the indentation behavior of the sandwich plates is verified by comparing them with finite element $(\mathrm{FE})$ predictions. To predict the indentation response of the sandwich plates, axisymmetric FE models were developed in ABAQUS CAE version 6.11. The computational geometry along with the loading and boundary conditions are shown in Figure 2. The faceplates and core were meshed using four node bilinear axisymmetric reduced integration elements (CAX4R) while the flat punch (FP) was modeled as an analytical rigid body. To understand the implications of the assumed analytical analogy, indentation simulations are also conducted using ring punch (RP). The core was meshed with 100 elements in the thickness direction and faceplate has 6 elements in thickness direction, while maintaining 900 elements in the radial direction for both faceplate and core. Stiffness hourglass control was used to avoid spurious energy modes resulting from reduced integration. Smooth displacement loading was applied to the punch to simulate the quasi-static 
loading condition. Friction less contact is defined between faceplate and rigid punch.

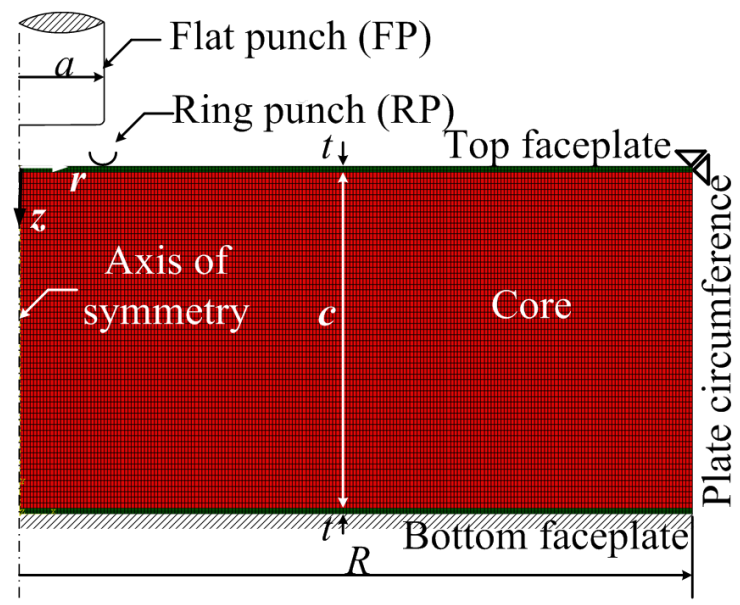

(a)

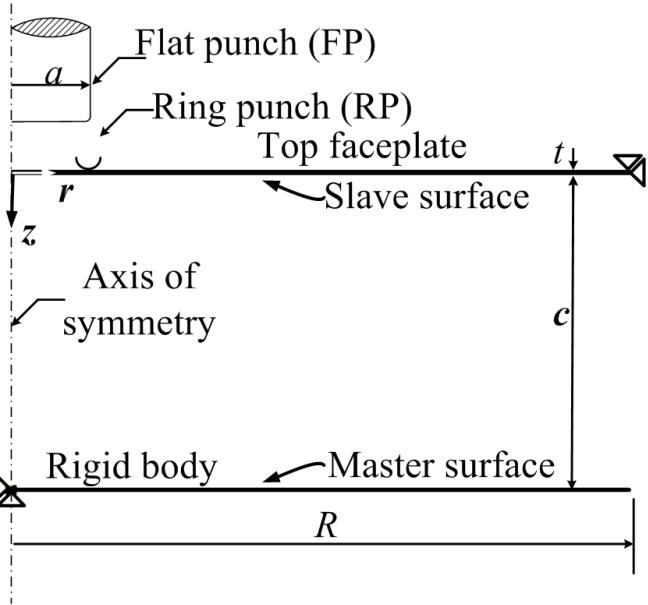

(b)

Figure 2 FE modelling strategies of the sandwich plate. (a) Continuum foam (CF) model and (b) Interaction (UINTER) model.

The core was modeled using two methodologies: (a) continuum foam $(\mathrm{CF})$ model in Figure 2a, and (b) interaction (UINTER) model in Figure 2b, In the CF model, the core was modeled using crushable foam constitutive model of Deshpande and Fleck [27]. Elastic properties of the foams are listed in Table 2. After the elastic limit, foam behavior is perfectly plastic with isotropic hardening. In the second methodology, i.e. UINTER model, the core and bottom faceplate are not modelled explicitly rather the reaction force (from the foam core) is applied on the top faceplate bottom surface (usually termed as a slave surface in interaction terminology). This is achieved using Abaqus user interaction subroutine UINTER with input variables $k$ and $\sigma_{c}$. To define the interaction, a dummy rigid surface (termed as master surface to represent bottom faceplate) is modeled to interact with a deformable slave surface. All degrees of freedom of the rigid master surface are constrained. Elastic foundation is simulated by applying the " $k w$ " pressure on the slave surface. In simulating the EPP foundation, if " $k w$ " is greater than $\sigma_{c}$ then a reaction pressure $\sigma_{c}$ is applied on the top faceplate. Hence, this is considered as a maximum (through-thickness) normal stress yield criterion, similar to the yield criterion used in the analytical modeling. 
To be consistent with the analytical formulations, small or large deformation theory simulations were carried out by switching the non-linear geometry (i.e. NLGEOM) option off or on, in Abaqus software.

Elastic foundation (CF and UINTER) and EPP (UINTER) simulations were carried out using implicit solver as UINTER subroutine can only be used with implicit solver. However, the EPP (CF) simulations were simulated using explicit solver.

\section{Results and discussion}

In this section, analytical stiffness and load-displacement estimates for the plate on elastic and EPP foundation are compared against the experimental measurements and FE predictions. Initially, the elastic solution is used as a benchmark solution to assess the stiffness prediction from different FE methodologies under consideration and validity of the assumption: FP imposes an axisymmetric line load. Later, the load-displacement estimates are compared against the FE predictions.

\subsection{Elastic foundation: stiffness response}

A comparison of stiffness estimates among different strategies are listed in Table 3. The maximum percentage difference between the flat punch (FP) and ring punch (RP) (in both $\mathrm{CF}$ and UINTER models) is $\approx 2.6 \%$, which decreases with increasing foam density. Hence, FP can be replaced with an axisymmetric line load.

Table 3 Comparison of stiffness $(\mathrm{N} / \mathrm{mm})$ prediction between different methodologies using indentation analogy.

\begin{tabular}{|c|c|c|c|c|c|c|c|c|c|c|c|}
\hline \multirow[b]{4}{*}{ Foam } & \multicolumn{11}{|c|}{ Methodology } \\
\hline & \multirow{3}{*}{$\begin{array}{l}\text { Anal. } \\
\text { Eq. }\end{array}$} & \multicolumn{10}{|c|}{$\mathrm{FE}$} \\
\hline & & \multicolumn{5}{|c|}{ Continuum foam } & \multicolumn{5}{|c|}{ UINTER } \\
\hline & & FP & error $^{\dagger}$ & RP & error $^{\ddagger}$ & error $^{\S}$ & FP & error $^{\dagger}$ & $\mathrm{RP}$ & error ${ }^{\dagger}$ & error $^{\S}$ \\
\hline H35 & 957.7 & 964.7 & -0.7 & 985.1 & -2.9 & -2.1 & 966.6 & -0.9 & 991.4 & -3.5 & -2.6 \\
\hline H45 & 1587.7 & 1472.6 & 7.2 & 1498.0 & 5.6 & -1.7 & 1604.3 & -1.0 & 1629.9 & -2.7 & -1.6 \\
\hline H80 & 2275.9 & 2004.7 & 11.9 & 2030.4 & 10.8 & -1.3 & 2329.3 & -2.3 & 2318.2 & -1.9 & 0.5 \\
\hline H100 & 2423.4 & 2117.4 & 12.6 & 2142.4 & 11.6 & -1.2 & 2489.0 & -2.7 & 2465.0 & -1.7 & 1.0 \\
\hline
\end{tabular}


The percentage error in stiffness between analytical estimates and FE predictions increases with increasing foam density. This error $(<10 \%)$ is due to the differences in representing the foam yield behavior in FE (CF) or loading condition in analytical modeling i.e. the load is considered to be axisymmetric line load. However, the predictions from RP model shows a decrease in percentage error with increasing foam density. Hence, it is evident that the analytical models are better validated using either FP/RP using UINTER models in the context of small deformation.

\subsection{Load versus displacement response}

The FE predictions (from UINTER model) of load variation with punch displacement shown in Figure 3 are in good agreement with the analytical estimates, as the deformation progresses beyond the core yield. Since the analytical stiffness solution for small deformation agreed well with the FE solutions of UINTER models rather than CF based models, in Figure 3 the FE predictions from CF models were not plotted for small deformations.

Small deformation (EPP) analytical estimates agreed well with the FE (small deformation) predictions up to $\delta / t$ of 4.0. However, these estimates were found to deviate from experimental measurements when $\delta / t>2$. This is due to large deformation (stretching) effect in the faceplates. The proposed analytical (RPP) estimates (with stretching) are well in agreement with the experiments and FE predictions up to the failure of the top faceplate both in FP as well as SP. Hosseini et al. [21] estimates are under predict load (at a given displacement), possibly due to the assumption of negligible midplane displacements. In the context of SP estimates, Zhou and Strong [12] estimates are too stiff and found to deviate far from the experimental measurements and hence omitted from the comparison. However, Türk and Hoo-Fatt [10] membrane estimates are also found to be stiff.

\subsection{Deflection profile}

The deflection profiles estimated from the analytical models are compared with the FE predictions in Figures 5 and 6 at a given indentation load for flat and spherical punches, 


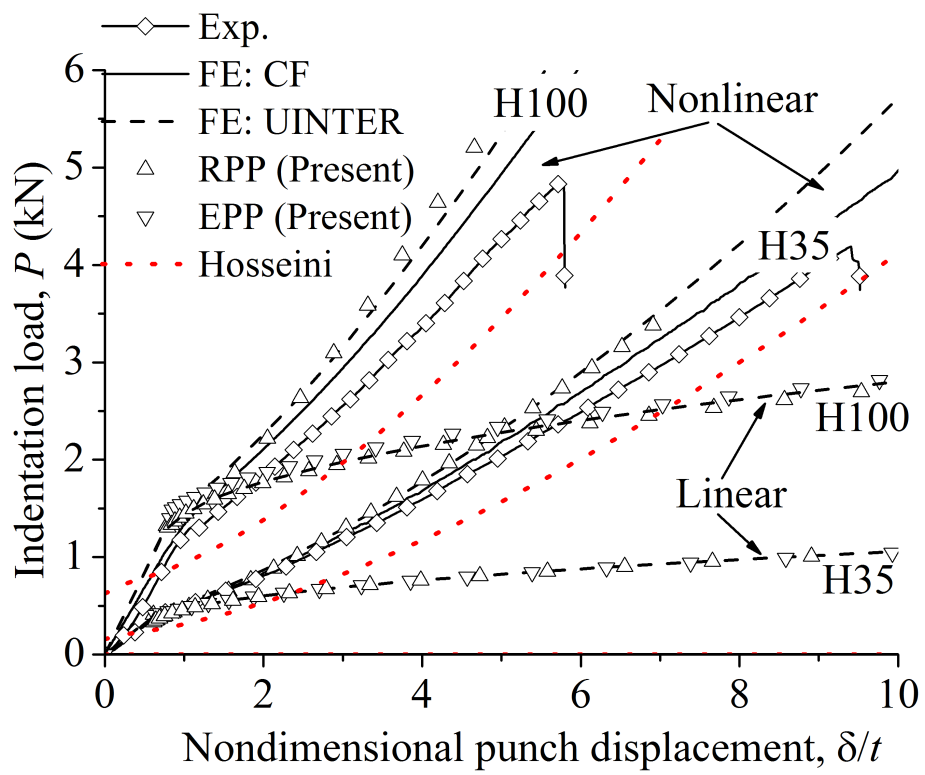

(a)

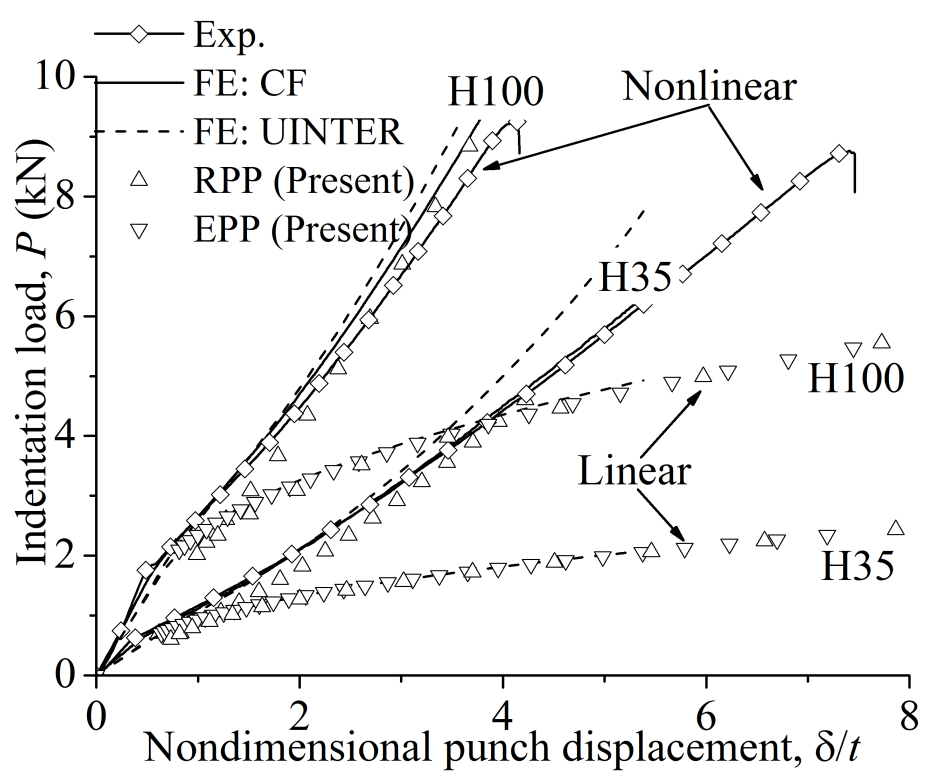

(b)

Figure 3 Indentation load versus nondimensional displacement responses for sandwich plates under FP loading with faceplate thickness (a) $t=0.7 \mathrm{~mm}$ and (b) $t=1.3 \mathrm{~mm}$. EPP, RPP-nonlinear and RPP-linear estimates from Eq. [5], Eq. (8) and Eq. [11a, respectively. Hosseini prediction is plotted from Hosseini et al.[21].

respectively. Experimental measurements of the deformation profile of the faceplate were not made during the tests, hence, the punch displacement is taken from the load-displacement curve and shown as a point. Analytical estimates of deformation profile for RPP foundation are offset by the elastic limit displacements $\left(\delta_{e l}\right.$ in Figures 5 and 6 ) to match with the EPP 


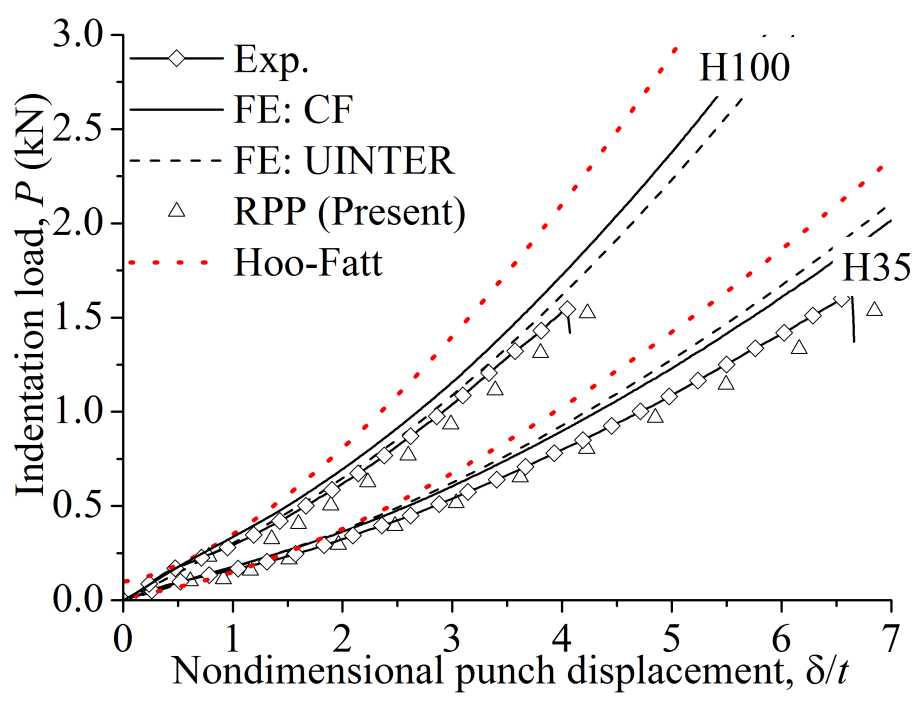

(a)

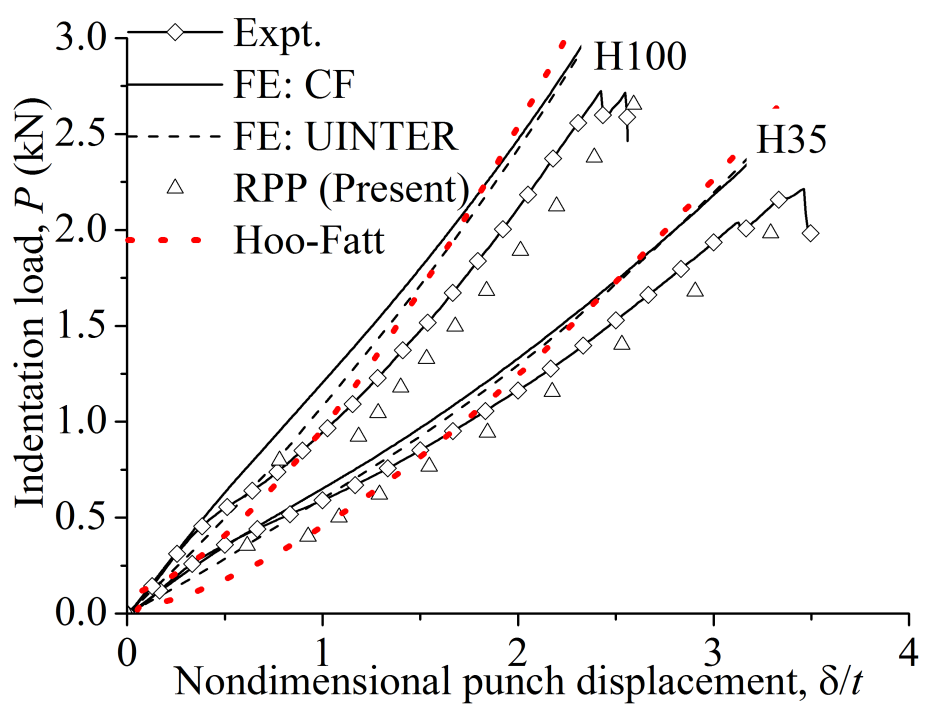

(b)

Figure 4 Indentation load versus nondimensional displacement responses for sandwich plates under SP loading with faceplate thickness (a) $t=0.7 \mathrm{~mm}$ and (b) $t=1.3 \mathrm{~mm}$. RPP estimate is from Eq. (13b). HooFatt curve is plotted from Türk and Hoo-Fatt [10].

foundation. This led to the discrepancy between the analytical estimates and FE predictions for $r \geq \lambda$. It is evident that the proposed analytical estimates are in better agreement with the FE predictions compared to the estimates of Hosseini et al. [21] and Türk and Hoo-Fatt [10]. 


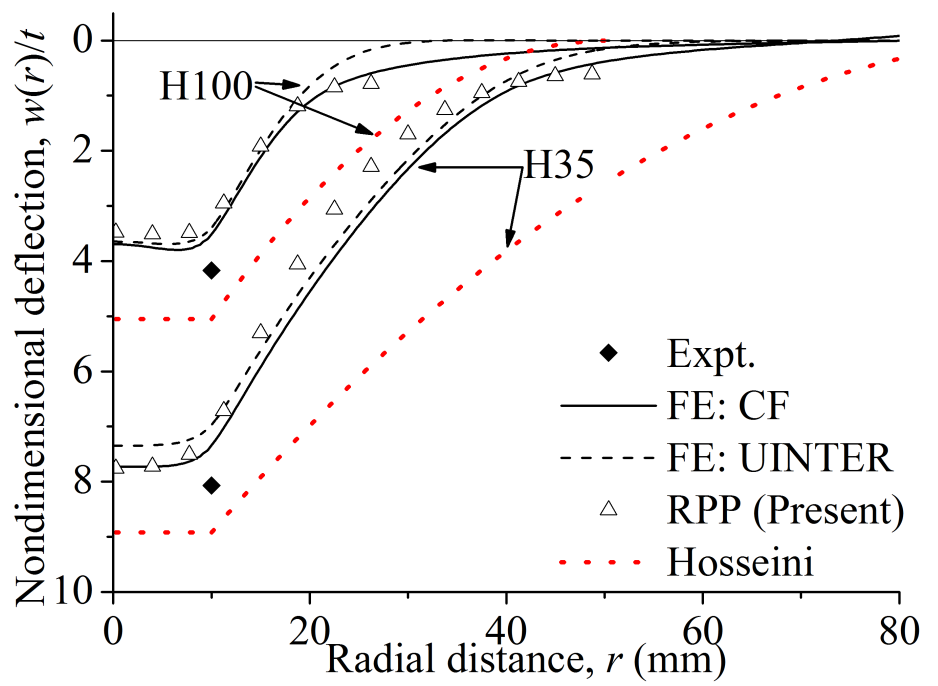

(a)

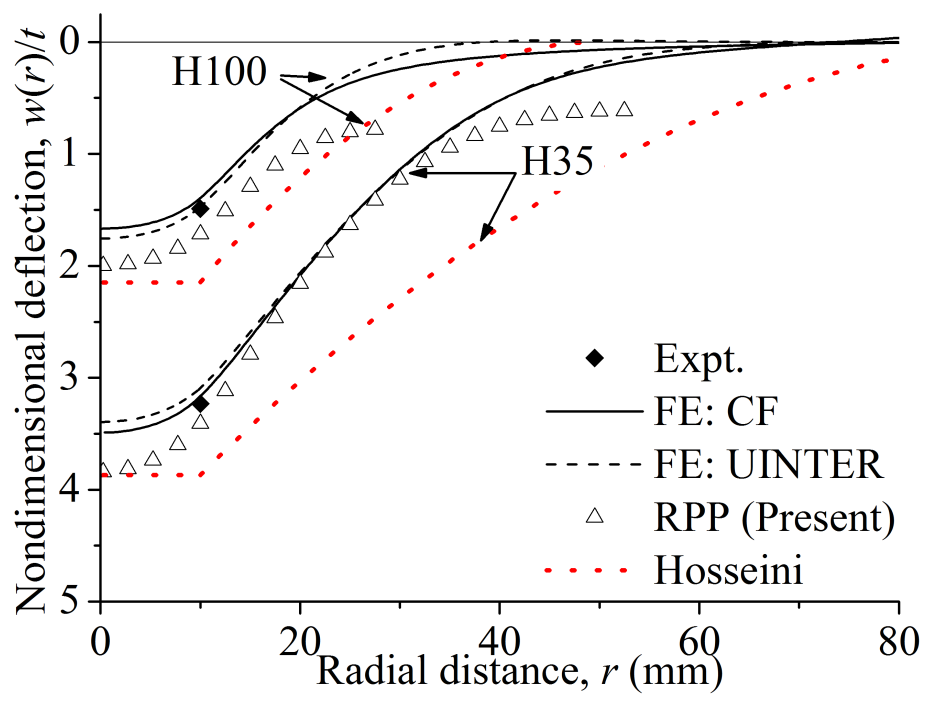

(b)

Figure 5 Comparison of deflection profiles by different formulations subjected to FP loading at $3.5 \mathrm{kN}$ indentation load with faceplate thickness (a) $t=0.7 \mathrm{~mm}$ and (b) $t=1.3 \mathrm{~mm}$. RPP estimate is from Eq. (8). Hosseini curve is plotted from Hosseini et al.[21].

\section{Conclusions}

Analytical formulations are proposed to estimate the indentation response of the composite sandwich plates subjected to quasi-static indentation by a rigid flat punch (FP). The faceplate was assumed to have linear elastic behavior with axisymmetric deformation and the indentation response has been modeled using beam on foundation methodology. The loading of flat punch (FP) is assumed to impose an axisymmetric line load on the faceplate along the 


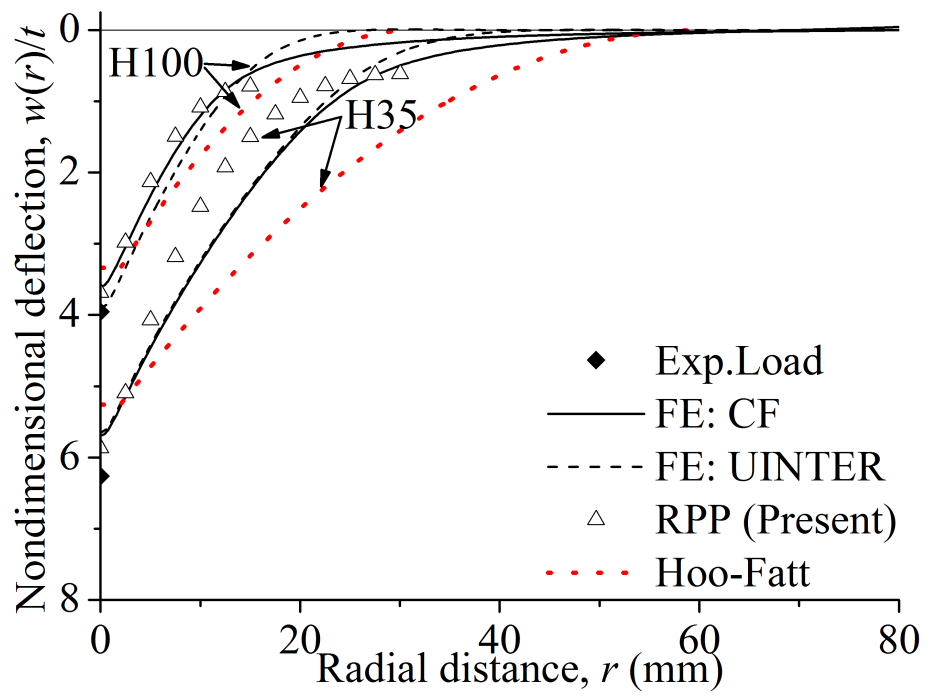

(a)

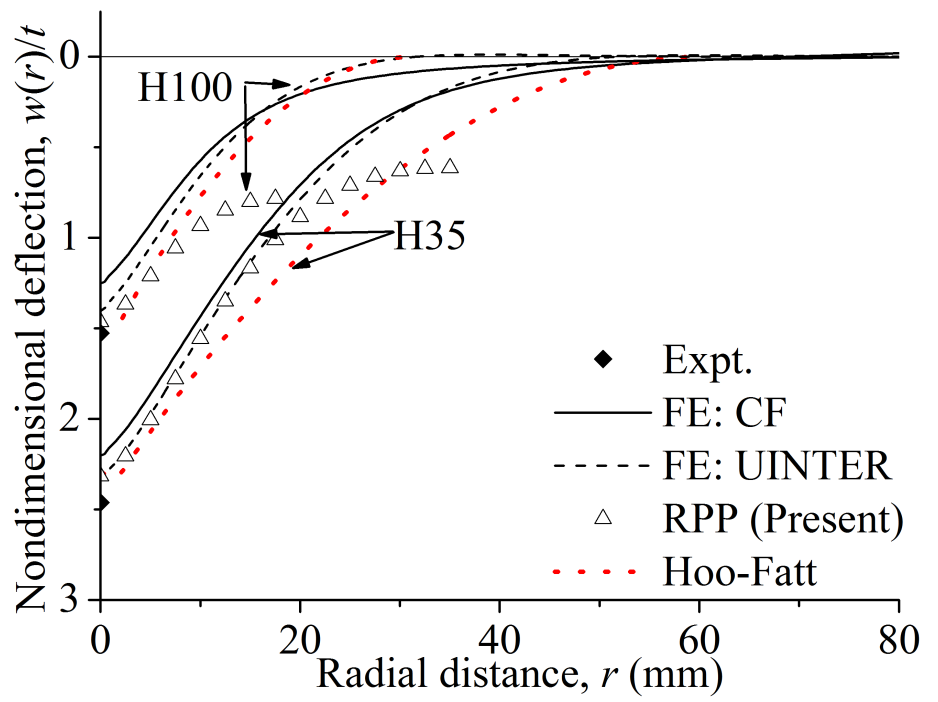

(b)

Figure 6 Comparison of deflection profiles by different formulations subjected to SP loading at $1.5 \mathrm{kN}$ indentation load with faceplate thickness (a) $t=0.7 \mathrm{~mm}$ and (b) $t=1.3 \mathrm{~mm}$. RPP estimate is from Eq. (13a). HooFatt curve is plotted from Türk and Hoo-Fatt [10].

punch radius, $a$.

Typical polymer and metallic foams show EPP behavior under uniaxial compression and hence the core is treated as an EPP foundation in the context of small deformations and the governing differential equation is solved exactly. However small deformation solutions deviate from the actual indentation loads when the indentation depth is greater than two times the thickness of the faceplate; due to the large deformation effects in the faceplate. 
Hence, Berger's approximate differential equations are considered for RPP foundation and the differential equations are solved exactly for FP loading. SP indentation response was derived as a limiting case: flat punch solution with zero radius. Derived RPP core loaddisplacement relations and deflection profiles were offset by elastic limit displacement, $\delta_{e l}$ (without altering the load) to achieve the EPP foundation response.

Reasonable agreement is observed among FE predictions, experimental measurements and analytical estimates for the indentation load-displacement and deflection profile of the top faceplate at applied loads that satisfy small/large deformation regimes.

\section{Acknowledgements}

A. Rajaneesh and A. R. Akisanya acknowledge the financial support from Nanyang Technological University, Singapore through award of Graduate Scholarship and Tan Chin Tuan (TCT) Visiting Fellowship, respectively.

\section{Appendix A Indentation of a plate on EPP foundation}

The coefficients in the displacement function Eq. (5) for a plate on elastic-perfectly plastic foundation based on indentation analogy are defined as follows.

$$
\begin{gathered}
B_{1}=B_{5}+B_{3}[\ln (a)+1] ; \quad B_{2}=B_{6}+B_{4}[\ln (a)-1] ; \quad B_{3}=\frac{P}{8 \pi D_{f}} ; \quad B_{4}=a^{2} B_{3} \\
B_{5}=\frac{\sigma_{c}}{4 D_{f} \eta_{12}}\left\{8 \lambda l^{3} \eta_{5}[\ln (\lambda)+1]+\frac{\lambda l \eta_{6}}{2}\left[4 \lambda^{2} \ln (\lambda)+\lambda^{2}+2 a^{2}\right]\right. \\
\left.-\sqrt{2} l^{2} \eta_{7}\left[4 \lambda^{2} \ln (\lambda)+2 \lambda^{2}+a^{2}\right]+\frac{\eta_{8}}{2 \sqrt{2}}\left[\lambda^{4} \ln (\lambda)+16 l^{4}+a^{2} \lambda^{2}\right]\right\} \\
B_{6}=\frac{\sigma_{c}}{64 D_{f} \eta_{12}}\left\{128 \lambda l^{3} \eta_{5}\left[a^{2} \ln (\lambda)-\lambda^{2}\right]\right. \\
\quad+4 \lambda l \eta_{6}\left[8 a^{2} \lambda^{2} \ln (\lambda)-3 \lambda^{4}-4 a^{2} \lambda^{2}-64 l^{4}\right] \\
-\sqrt{2} l^{2} \eta_{7}\left[64 a^{2} \lambda^{2} \ln (\lambda)-36 \lambda^{4}-16 a^{2} \lambda^{2}-256 l^{4}\right] \\
\left.-\sqrt{2} \eta_{8}\left[a^{2} \lambda^{4}(3-4 \ln (\lambda))+\lambda^{6}+128 l^{4} \lambda^{2}-64 a^{2} l^{4}\right]\right\} \\
B_{7}=\frac{\sigma_{c} l^{3}}{2 D_{f} \eta_{13}}\left\{2 \sqrt{2} l\left[4 \eta_{2} l^{2}-\eta_{1}\left(\lambda^{2}-a^{2}\right)\right]-8 \lambda l^{2} f_{0}(\lambda)+\lambda g_{0}(\lambda)\left(2 a^{2}-\lambda^{2}\right)\right\}
\end{gathered}
$$




$$
B_{8}=-\frac{\sigma_{c} l^{3}}{2 D_{f} \eta_{12}}\left\{2 \sqrt{2} l\left[4 \eta_{1} l^{2}+\eta_{2}\left(\lambda^{2}-a^{2}\right)\right]+8 \lambda l^{2} g_{0}(\lambda)+\lambda f_{0}(\lambda)\left(2 a^{2}-\lambda^{2}\right)\right\}
$$

where

$$
\begin{array}{lll}
\eta_{1}=\mathrm{f}_{1}(\lambda)-\mathrm{g}_{1}(\lambda) ; & \eta_{2}=\mathrm{f}_{1}(\lambda)+\mathrm{g}_{1}(\lambda) ; & \eta_{3}=\mathrm{f}_{0}(\lambda)-\mathrm{g}_{0}(\lambda) \\
\eta_{4}=\mathrm{f}_{0}(\lambda)+\mathrm{g}_{0}(\lambda) ; & \eta_{5}=\left[\mathrm{f}_{1}(\lambda)\right]^{2}+\left[\mathrm{g}_{1}(\lambda)\right]^{2} ; & \eta_{6}=\left[\mathrm{f}_{0}(\lambda)\right]^{2}+\left[\mathrm{g}_{0}(\lambda)\right]^{2} \\
\eta_{7}=\eta_{2} \mathrm{f}_{0}(\lambda)-\eta_{1} \mathrm{~g}_{0}(\lambda) ; & \eta_{8}=\eta_{1} \mathrm{f}_{0}(\lambda)+\eta_{2} \mathrm{~g}_{0}(\lambda) ; & \eta_{9}=\eta_{3} \mathrm{~g}_{1}(\lambda)-\eta_{4} \mathrm{f}_{1}(\lambda) \\
\eta_{10}=\eta_{3} \mathrm{f}_{1}(\lambda)+\eta_{4} \mathrm{~g}_{1}(\lambda) ; & \eta_{11}=\frac{P_{e l}^{i}}{2 \pi}-\frac{\sigma_{c} \lambda^{2}}{2} & \\
\eta_{12}=\sqrt{2}\left[4 \eta_{7} l^{2}-\eta_{8}\left(\lambda^{2}-a^{2}\right)\right]-4 l \lambda \eta_{6} & \\
\eta_{13}=\sqrt{2}\left[4 \eta_{10} l^{2}+\eta_{9}\left(\lambda^{2}-a^{2}\right)\right]-4 l \lambda \eta_{6} &
\end{array}
$$

\section{Appendix B Indentation of a plate on RPP with FP}

Integration constants in Eq. (8) are

$$
\begin{array}{ll}
C_{1}=\frac{P\left(1-\Gamma_{1}\right)}{2 \pi D_{f} \alpha^{3} \lambda \mathrm{I}_{1}(\alpha \lambda)}-\frac{\sigma_{c} \lambda}{2 D_{f} \alpha^{3} \mathrm{I}_{1}(\alpha \lambda)} & \\
C_{2}=\frac{P}{2 \pi D_{f} \alpha^{3} \lambda}\left[\frac{\mathrm{I}_{0}(\alpha a)-\mathrm{I}_{0}(\alpha \lambda)}{\mathrm{I}_{1}(\alpha \lambda)}+\alpha \lambda \ln \left(\frac{\lambda}{a}\right)\right]+\frac{\sigma_{c} \lambda}{4 D_{f} \alpha^{3} \lambda}\left[\frac{2 \mathrm{I}_{0}(\alpha \lambda)}{\mathrm{I}_{1}(\alpha \lambda)}-\alpha \lambda\right] \\
\Gamma_{1}=\alpha \lambda\left[\mathrm{I}_{1}(\alpha \lambda) \mathrm{K}_{0}(\alpha a)+\mathrm{K}_{1}(\alpha \lambda) \mathrm{I}_{0}(\alpha a)\right] ; & \Upsilon_{1}=\mathrm{I}_{0}(\alpha r) \mathrm{K}_{0}(\alpha a)-\mathrm{K}_{0}(\alpha r) \mathrm{I}_{0}(\alpha a) \\
\Upsilon_{2}=\mathrm{K}_{1}(\alpha r)^{2}-\mathrm{K}_{0}(\alpha r) \mathrm{K}_{2}(\alpha r) ; & \Upsilon_{3}=\mathrm{I}_{1}(\alpha r)^{2}-\mathrm{I}_{0}(\alpha r) \mathrm{I}_{2}(\alpha r) \\
\Upsilon_{4}=r G_{2,4}^{2,2}\left(\begin{array}{c}
0, \frac{1}{2} \\
0,1,-1,-1
\end{array} \mid \alpha^{2} r^{2}\right) ; & \Upsilon_{5}=\mathrm{I}_{0}(\alpha a) \mathrm{K}_{2}(\alpha r)-\mathrm{K}_{0}(\alpha a) \mathrm{I}_{2}(\alpha r) \\
\Upsilon_{6}=\frac{1}{16 \pi^{2} r D_{f}^{2} \alpha^{4}}\left\{\alpha^{2} r^{2}\left[\mathrm{I}_{0}(\alpha a)^{2} \Upsilon_{2}+\mathrm{K}_{0}(\alpha a)^{2} \Upsilon_{3}\right]-4 \Upsilon_{1}+2 \ln (r)\right\} \\
\Upsilon_{7}=\frac{C_{1} \mathrm{~K}_{0}(\alpha a) r \Upsilon_{3}}{4 \pi D_{f}}-\frac{C_{1} \mathrm{I}_{0}(\alpha r)}{2 \pi \alpha^{2} r D_{f}} &
\end{array}
$$

where $G$ is the Meijer-G function. 


\section{Appendix C Indentation of a plate on RPP with SP}

$$
\begin{aligned}
& F_{1}=\frac{P\left(1-\Gamma_{1}^{\prime}\right)}{2 \pi D_{f} \alpha^{3} \lambda \mathrm{I}_{1}(\alpha \lambda)}-\frac{\sigma_{c} \lambda}{2 D_{f} \alpha^{3} \mathrm{I}_{1}(\alpha \lambda)} \\
& F_{2}=\frac{P}{2 \pi D_{f} \alpha^{3} \lambda}\left[\frac{\mathrm{I}_{0}(\alpha a)-\mathrm{I}_{0}(\alpha \lambda)}{\mathrm{I}_{1}(\alpha \lambda)}+\alpha \lambda \ln (\lambda)\right]+\frac{\sigma_{c} \lambda}{4 D_{f} \alpha^{3} \lambda}\left[\frac{2 \mathrm{I}_{0}(\alpha \lambda)}{\mathrm{I}_{1}(\alpha \lambda)}-\alpha \lambda\right] \\
& F_{3}=-\frac{P^{2}}{8 \pi^{2} \alpha^{4} D_{f}^{2}}\left[\ln \left(\frac{\alpha}{2}\right)+\gamma-\frac{1}{2}\right]-\frac{P \sigma_{c}}{2 \pi \alpha^{6} D_{f}^{2}}-\frac{P F_{1}}{2 \pi \alpha^{2} D_{f}}
\end{aligned}
$$

where $\gamma=0.577$ is the Euler's constant.

$$
\begin{array}{lll}
\Gamma_{1}^{\prime}=\alpha \lambda \mathrm{K}_{1}(\alpha \lambda) ; & \Upsilon_{1}^{\prime}=-\mathrm{K}_{0}(\alpha r) ; & \Upsilon_{2}^{\prime}=\mathrm{K}_{1}(\alpha r)^{2}-\mathrm{K}_{0}(\alpha r) \mathrm{K}_{2}(\alpha r) \\
\Upsilon_{3}^{\prime}=\mathrm{I}_{1}(\alpha r)^{2}-\mathrm{I}_{0}(\alpha r) \mathrm{I}_{2}(\alpha r) ; & \Upsilon_{4}^{\prime}=r G_{2,4}^{2,2}\left(\begin{array}{c}
0, \frac{1}{2} \\
0,1,-1,-1
\end{array} \mid \alpha^{2} r^{2}\right) ; & \Upsilon_{5}^{\prime}=\mathrm{K}_{2}(\alpha r) \\
\Upsilon_{6}^{\prime}=\frac{1}{16 \pi^{2} r D_{f}^{2} \alpha^{4}}\left[\alpha^{2} r^{2} \Upsilon_{2}^{\prime}-4 \Upsilon_{1}^{\prime}+2 \ln (r)\right] ; & \Upsilon_{7}^{\prime}=-\frac{F_{1} \mathrm{I}_{0}(\alpha r)}{2 \pi \alpha^{2} r D_{f}}
\end{array}
$$

\section{References}

[1] M. M. Sommer, M. Päßler, and R. Schledjewski. Sandwich Structure for Sports Sticks. Kunststoffe international, May/2004.

[2] Evonik Industries. Cross-Country Skiing Made Easy. Product Story No. 30, February, 2010.

[3] A. Ashab, D. Ruan, G. Lu, S. Xu, and C. Wen. Experimental investigation of the mechanical behavior of aluminum honeycombs under quasi-static and dynamic indentation. Materials \& Design, 74(0):138-149, 2015.

[4] H. Mozafari, S. Khatami, and H. Molatefi. Out of plane crushing and local stiffness determination of proposed foam filled sandwich panel for korean tilting train expressnumerical study. Materials \& Design, 66, Part B(0):400 - 411, 2015.

[5] C. H. Lim, I. Jeon, and K. J. Kang. A new type of sandwich panel with periodic cellular metal cores and its mechanical performances. Materials \& Design, 30(8):3082 - 3093, 2009. 
[6] G. Di Bella, C. Borsellino, and L. Calabrese. Effects of manufacturing procedure on unsymmetrical sandwich structures under static load conditions. Materials \& Design, 35(0):457 - 466, 2012.

[7] P. Navarro, S. Abrate, J. Aubry, S. Marguet, and J. F. Ferrero. Analytical modeling of indentation of composite sandwich beam. Composite Structures, 100(0):79-88, 2013.

[8] Y. Frostig, O. T. Thomsen, and I. Sheinman. On the non-linear high-order theory of unidirectional sandwich panels with a transversely flexible core. International Journal of Solids and Structures, 42(5-6):1443-1463, 2005.

[9] R. Olsson and H. L. McManus. Improved theory for contact indentation of sandwich panels. AIAA journal, 34(6):1238-1244, 1996.

[10] M. H. Türk and M. S. Hoo-Fatt. Localized damage response of composite sandwich plates. Composites Part B: Engineering, 30(2):157-165, 1999.

[11] V. Koissin, A. Shipsha, and V. Rizov. The inelastic quasi-static response of sandwich structures to local loading. Composite Structures, 64(2):129-138, 2004.

[12] D. W. Zhou and W. J. Stronge. Low velocity impact denting of HSSA lightweight sandwich panel. International Journal of Mechanical Sciences, 48(10):1031-1045, 2006.

[13] V. Z. Vlasov and N. N. Leont'ev. Beams, Plates and Shells on Elastic Foundations. Israel Program for Scientific Translations, Jerusalem, 1966.

[14] S. Timoshenko and S. Woinowsky-Krieger. Theory of Plates and Shells. Engineering societies monographs. McGraw-Hill, USA, 2nd edition, 1970.

[15] M. Sathyamoorthy. Nonlinear analysis of structures. CRC Press, USA, 1997.

[16] O. T. Thomsen. Theoretical and experimental investigation of local bending effects in sandwich plates. Composite Structures, 30(1):85-101, 1995. 
[17] T. Anderson and E. Madenci. Graphite/epoxy foam sandwich panels under quasi-static indentation. Engineering Fracture Mechanics, 67(4):329-344, 2000.

[18] R. Sburlati. The contact behavior between a foam core sandwich plate and a rigid indentor. Composites Part B: Engineering, 33(4):325-332, 2002.

[19] C. Y. Chia. Nonlinear Analysis of Plates. McGraw-Hill, USA, 1980.

[20] O. T. Thomsen. Analysis of local bending effects in sandwich plates with orthotropic face layers subjected to localised loads. Composite Structures, 25(1-4):511-520, 1993.

[21] M. Hosseini, S. M. R. Khalili, and K. Malekzadeh Fard. An indentation law for doubly curved composite sandwich panels with rigid-plastic core subjected to flat-ended cylindrical indenters. Composite Structures, 105(0):82-89, 2013.

[22] H. M Berger. A new approach to the analysis of large deflections of plates. $\mathrm{PhD}$ Thesis, California Institute of Technology, (USA), 1954.

[23] T. Vodenitcharova, K. Kabir, and M. Hoffman. Indentation of metallic foam core sandwich panels with soft aluminium face sheets. Materials Science and Engineering: A, 558:175-185, 2012.

[24] Z. Xie, Z. Zheng, and J. Yu. Localized indentation of sandwich panels with metallic foam core: Analytical models for two types of indenters. Composites Part B: Engineering, 44(1):212-217, 2013.

[25] A. Rajaneesh, I. Sridhar, and S. Rajendran. Failure mode maps for circular composites sandwich plates under bending. International Journal of Mechanical Sciences, 83:184$195,2014$.

[26] V. Panc. Theories of Elastic Plates. Noordhoff International, Netherlands, 1975. 
[27] V. S. Deshpande and N. A. Fleck. Isotropic constitutive models for metallic foams. Journal of the Mechanics and Physics of Solids, 48(6-7):1253-1283, 2000. 